\title{
Acute Heart Failure after Oral Intake of Liquid Nicotine
}

\author{
Young Shin Cho, Youngwha Sohn \\ Department of Emergency Medicine, Soonchunhyang University Seoul Hospital, Seoul, Korea
}

\begin{abstract}
A 32-year-old female patient was admitted to the hospital after drinking $10 \mathrm{mg}$ of liquid nicotine. She complained of dizziness, nausea, and abdominal pain. The blood pressure was low but the heart rate and respiratory rate were within a normal range. Cardiac enzymes were increased. Echocardiography taken in the emergency room showed akinesia in the apex and the anterior wall, hypokinesia in the inferior and the posterior wall, and severe left ventricular systolic dysfunction with an ejection fraction of $20 \%$. Blood pressure was continuously low after hydration so continuous intravenous norepinephrine and dobutamine were administrated. The patient was admitted to the intensive care unit for closed monitoring. Follow-up echocardiography was performed 4 days after admission. The heart was restored and the ejection fraction was recovered to $65 \%$. There are not many studies about the toxicity of electronic cigarette's nicotine and there are even fewer reports on the toxicity that occurs when liquid nicotine is orally taken. This is a report of a patient of an acute heart failure after intake of liquid nicotine used in the electronic cigarette.
\end{abstract}

Keywords: Heart failure; Nicotine; Echocardiography

\section{INTRODUCTION}

Electronic cigarettes (e-cigarettes) are a handheld electronic device that dispenses nicotine in the form of aerosol [1]. People using e-cigarettes are increasing daily because of stricter laws for cigarette smoking in public areas, lesser smell, lesser toxic substances, and no ashes compared to a traditional cigarette. E-cigarette shops are also increasing which allows the users to easily obtain the substance. Nicotine is an alkaloid that stimulates the central nervous system and autonomic ganglia through multiple molecular and cellular mechanisms [2]. When nicotine binds to its postsynaptic nicotinic acetylcholine receptor in the brain, ligand-gated ion channels open, allowing cation influx and eventual depolarization of the postsynaptic neuron and central nervous system excitation [2]. Although binding of nicotinic acetylcholine receptor predominantly yields sympathetic nervous stimulation, excess or prolonged exposure to nicotine may result in loss of receptor specificity or even paradoxical receptor blockade. Subsequent parasympathetic effects, cholinergic toxicity, and depolarizing neuromuscular blockage may result [2]. Pharmacokinetics after oral ingestion of liquid nicotine are not yet well established, although mucosal absorption has been shown to increase at alkaline $\mathrm{pH}$ [2]. In particular, there are even fewer reports on the toxicity that occurs when liquid nicotine is orally taken. In this study, we present a case of acute heart failure after ingestion liquid nicotine used in ecigarettes.

\section{CASE REPORT}

A 32-year-old female patient was admitted to the emergency room with dizziness, nausea, abdominal pain, and general weakness after taking $10 \mathrm{mg}$ of liquid nicotine used in e-cigarettes, 4 hours prior to the visit. The patient had a past history of depression and anxiety diagnosed 4 months prior and was taking oral medication prescribed by the primary neuropsychiatry. According to her statement, she drank $10 \mathrm{mg}$ of liquid nicotine mixed with 2 glasses of soju and a glass of beer to commit suicide because she felt depressed.

At the time of admission, her conscious level was drowsy but no other abnormal findings were observed in neurological examinations. In the vital signs, blood pressure was $78 / 57 \mathrm{~mm} \mathrm{Hg}$, pulse rate 88 beats per minute, respiratory rate 11 breaths per minute, 
and body temperature was $36.6^{\circ} \mathrm{C}$. On laboratory tests, white blood cell level was $16,700 / \mu \mathrm{L}$, hemoglobin was $13.5 \mathrm{~g} / \mathrm{dL}$, hematocrit $41.0 \%$, and platelet count was $388,000 / \mu \mathrm{L}$. The cardiac enzymes were increased. Creatine kinase myocardial bound (CK$\mathrm{MB}$ ) was $7.91 \mathrm{ng} / \mathrm{mL}$ (reference range, $0-5.8 \mathrm{ng} / \mathrm{mL}$ ), myoglobin was $77.49 \mathrm{ng} / \mathrm{mL}$ (reference range, $27-75 \mathrm{ng} / \mathrm{mL}$ ), and troponin $\mathrm{T}$ was $0.266 \mathrm{ng} / \mathrm{mL}$ (reference range, $0-0.1 \mathrm{ng} / \mathrm{mL}$ ). There was no evidence of pulmonary edema on chest radiography. Also, there was no specific finding on the brain computed tomography scan. Electrocardiogram showed QT prolongation (QTc 541) and nonspecific ST-T segment change (Fig. 1). Echocardiography taken in the emergency room showed akinesia in the apex and the anterior wall, hypokinesia in the inferior and the posterior wall, and severe left ventricular (LV) systolic dysfunction with an ejection fraction of $20 \%$ (Fig. 2). Gastric lavage was conducted and activated charcoal was administered. Blood pressure was continuously low after $1 \mathrm{~L}$ hydration; therefore, we decided to insert a central venous line. Central venous pressure measured $9 \mathrm{~mm} \mathrm{Hg}$ and inferior vena cava diameter was $2.2 \mathrm{~cm}$ without a plethora. Even with the ad- ministration of continuous intravenous norepinephrine, the blood pressure decreased to $62 / 48 \mathrm{~mm} \mathrm{Hg}$; therefore, continuous intravenous dobutamine was administrated. The patient was referred to the cardiology department due to acute heart failure caused by nicotine. The patient was admitted to the intensive care unit for closed monitoring.

The patient's consciousness was restored within the first day of admission. On the 4 th day of admission, the laboratory test showed a decrease in cardiac enzymes which were $2.74 \mathrm{ng} / \mathrm{mL}$ for CK-MB, $<21 \mathrm{ng} / \mathrm{mL}$ for myoglobin, and $0.111 \mathrm{ng} / \mathrm{mL}$ for troponin T. On the first day of admission, we performed a cotinine test. A few days later we were able to see the results. The amount of cotinine in serum was also increased to $7,069 \mathrm{ng} / \mathrm{mL}$. On follow-up echocardiography, the function of the anterior, inferior, and posterior walls of the heart was restored and only the apex showed hypokinesia (Fig. 3). Also, the ejection fraction was recovered to $65 \%$. The patient was discharged after the recovery of symptoms.

The patient provided written informed consent for publication of the research details and clinical images.

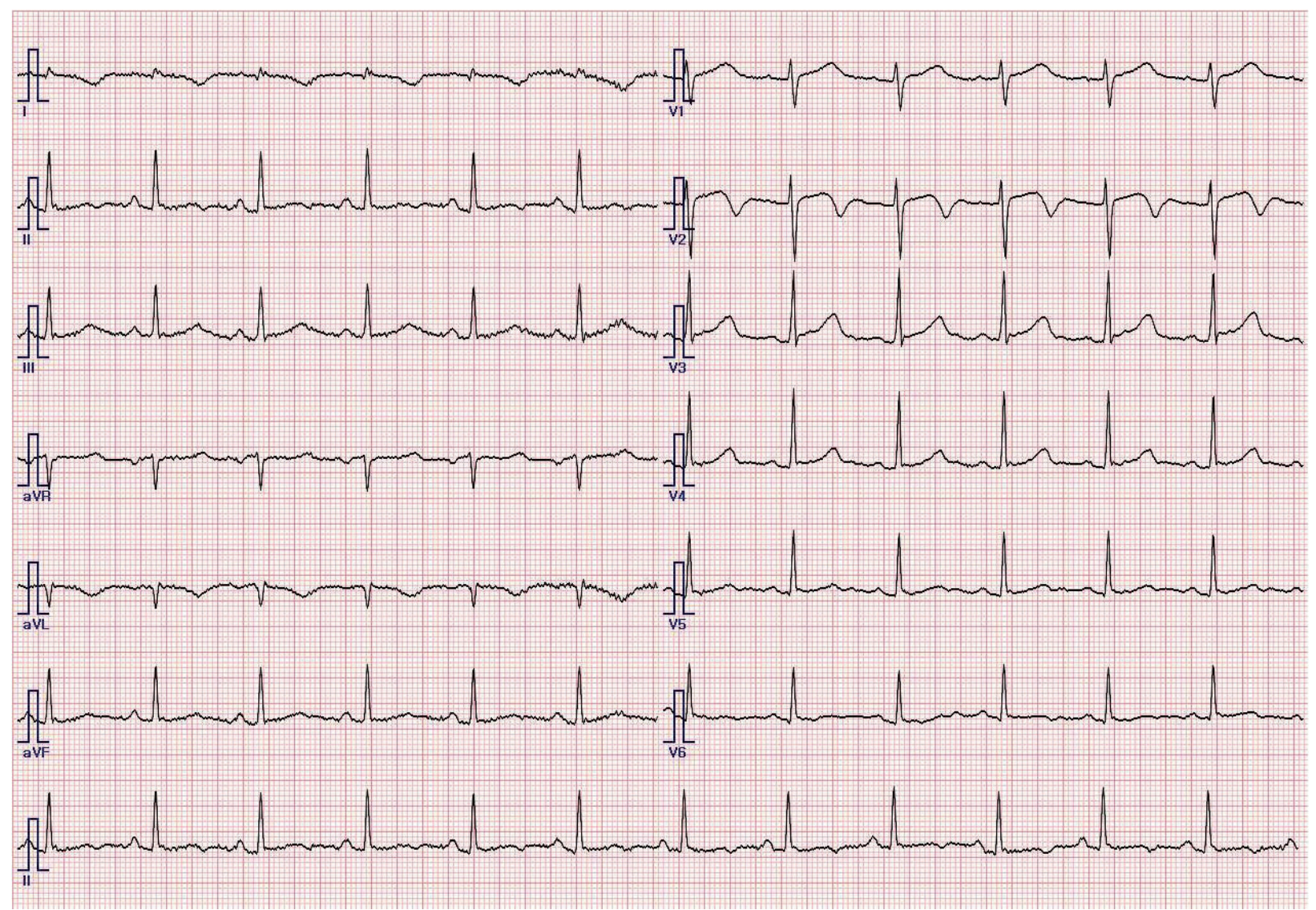

Fig. 1. The electrocardiography. Lead I and aVL presented T wave inversion but there were no ST elevations. 

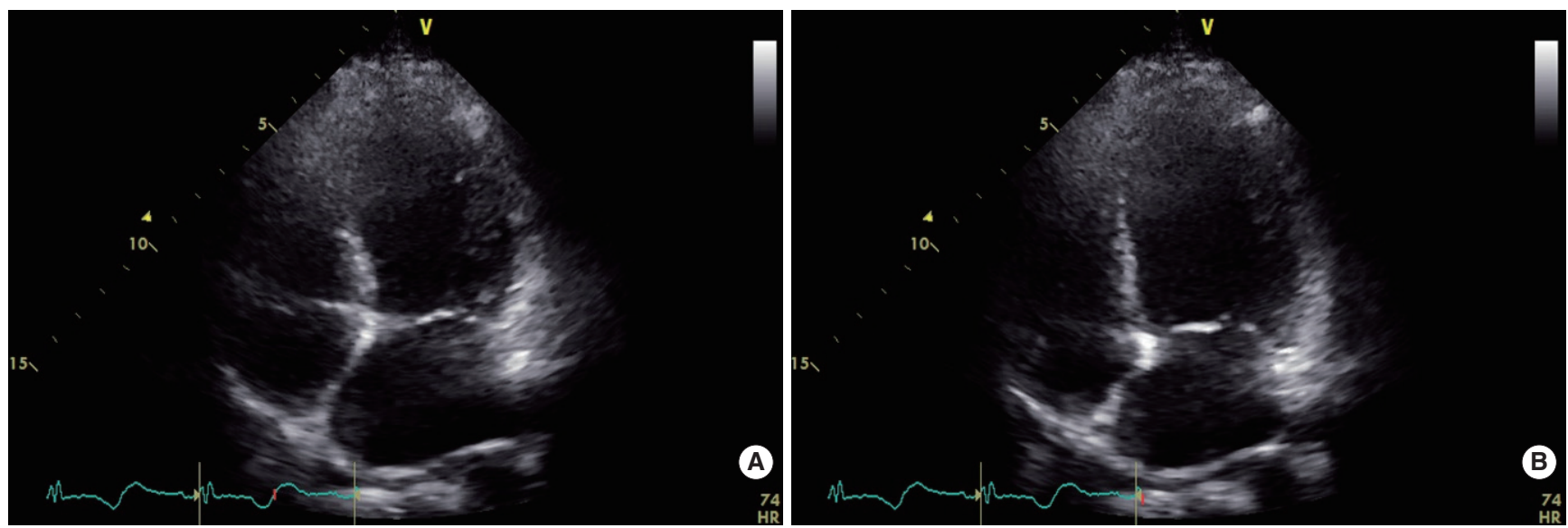

Fig. 2. Initial echocardiography (apical 4 chamber view). (A) End systole. (B) End diastole. It presented akinesia in the apex and anterior wall and hypokinesia in the inferior and decreased left ventricular systolic function.
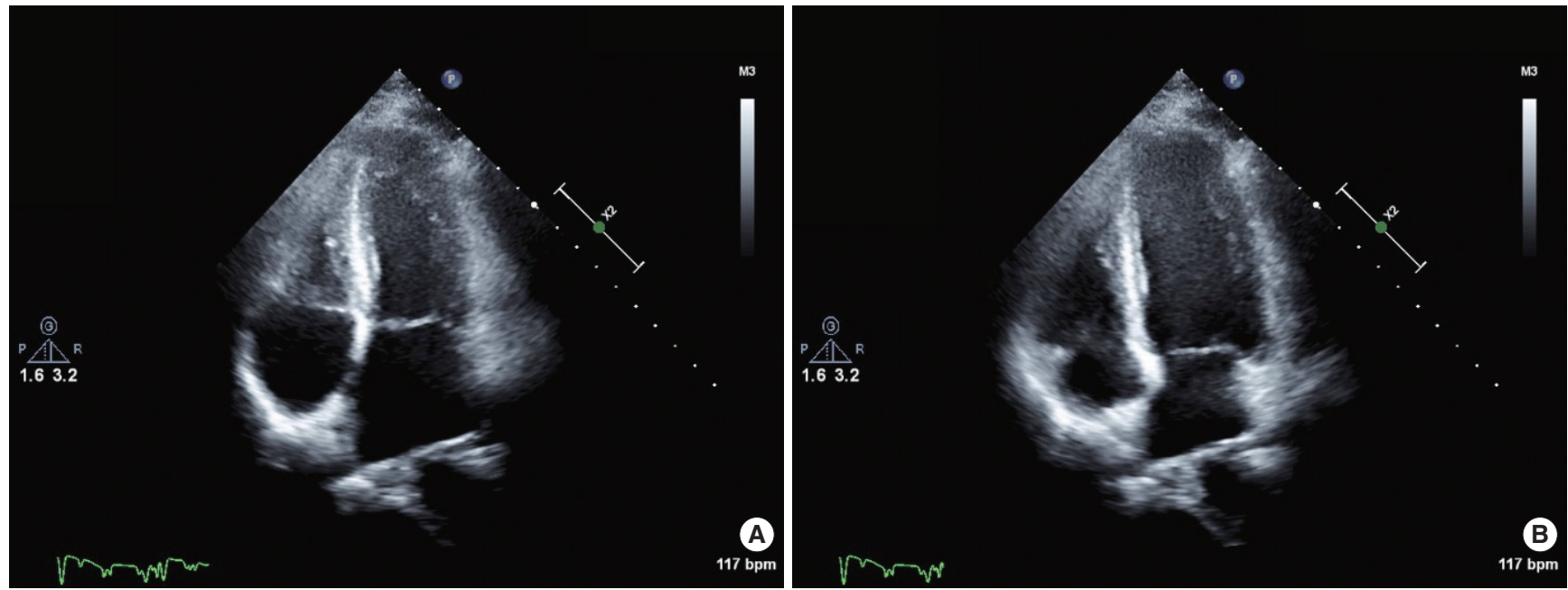

Fig. 3. Follow-up echocardiography on the 4th day of admission (apical 4 chamber view). (A) End systole. (B) End diastole. The wall motion abnormality of left ventricle was restored and ejection fraction was recovered.

\section{DISCUSSION}

We present a case of acute heart failure following intentional nicotine ingestion. Nicotine is an alkaloid that causes many biological effects, the intensity of which depends on the dosage [1]. It shows effects through nicotinic receptors, located mainly in the autonomic nervous system. Depending on the concentration, low doses have a stimulating effect, while high doses block nicotine receptors [1].

The overdose of nicotine has symptoms of the digestive system such as nausea, vomiting, and diarrhea which appear within a few minutes and systemic reactions such as anxiety, headache, sweating, hallucinations, hypertension, bradycardia, and tachycardia may occur. In severe poisoning, muscle weakening can lead to respiratory muscle paralysis and death $[1,3]$. Seizures and arrhythmia may occur several hours after intake, so observation for a few hours is necessary. Fatal dose of nicotine for adults is estimated to be $40-60 \mathrm{mg}$ or $0.8-1.0 \mathrm{mg} / \mathrm{kg}$ [4]. The peak plasma concentrations are usually reported 1 hour after oral administration. Nicotine metabolism mainly takes place in the liver. The plasma halflife of nicotine is $100-150$ minutes, and that of cotinine is 770 1,130 minutes [4].

Nicotine intoxication occurs through oral ingestion, smoking, and transdermal patch. In some cases, there are cases of intravenous poisoning $[1,3,5]$. The bioavailability of nicotine is significantly lower after oral ingestion than after smoking or application 
of transdermal nicotine patches, presumably due to the hepatic first-pass effect [4]. However, even if oral ingestion was performed, deaths occurred when taking lethal dose $[1,3,4,6,7]$.

There was another case report of acute myocardial infarction due to liquid nicotine poisoning in a young man [8]. There was STsegment elevation on electrocardiography and cardiac enzymes were increased. He was transferred to the cardiac catheterization laboratory, which revealed thrombus in the proximal left anterior descending artery [8]. Nicotine has a toxic effect on the endothelium, which may change its vascular activity and cause vasospasm, leading to myocardial infarction, as well as cause increased platelet aggregation [9].

In our case, the patient had drowsy mental status and complained of dizziness, nausea, and general weakness, but no chest pain and discomfort. Also, she presented low blood pressure without tachycardia or bradycardia. Although the ingested nicotine dose was not fatal, cardiac enzymes were increased and heart function was impaired. When we performed the echocardiography in the emergency room, there were regional wall motion abnormalities that did not match coronary artery territory. Also, there was no sign of stress-induced cardiomyopathy. There was severe LV systolic dysfunction with decreased ejection fraction. Cigarette smoking and smokeless tobacco use have been associated with an increased risk of heart failure [10]. Heart failure occurs in the context of tissue remodeling. In our case, the remodeling of the tissue did not proceed, but acute heart failure appeared. Because nicotine-induced heart failure can occur, adequate treatment of heart failure should be considered.

To date, there are no known antidotes against nicotine poisoning. Treatment of nicotine overdose generally consists of gastric lavage with the use of activated charcoal and ventilation or oxygenation therapy. As seizures or cardiac dysrhythmias may occur, observation of the patient for several hours is always required [4]. If heart failure occurs as in our case, it is helpful to supportive treatment that supports in contractility of the heart.

The market for e-cigarettes is growing annually and it is easily bought in a store nearby. Recently, it appears that suicidal nicotine poisoning is being shared through internet sites. There was a case report of suicidal nicotine poisoning using tobacco extract by in- ternet suicide guidelines [4]. Consequently, emergency physicians should anticipate a continued increase in cases of e-liquid exposures and poisonings.

In conclusion, as e-cigarettes are becoming popular and easily available, more patients with liquid nicotine overdose can occur. Therefore, we expected that emergency physicians will continue to encounter clinically significant cases of nicotine toxicity. Nicotine intoxication can cause acute heart failure with severe systolic dysfunction, so close observation and conservative treatment are needed.

\section{ACKNOWLEDGMENTS}

This work was supported by Soonchunhyang University Research Fund.

\section{REFERENCES}

1. Sommerfeld K, Lukasik-Glebocka M, Kulza M, Druzdz A, Panienski P, Florek E, et al. Intravenous and oral suicidal e-liquid poisonings with confirmed nicotine and cotinine concentrations. Forensic Sci Int 2016; 262:e15-20.

2. Noble MJ, Longstreet B, Hendrickson RG, Gerona R. Unintentional pediatric ingestion of electronic cigarette nicotine refill liquid necessitating intubation. Ann Emerg Med 2017;69:94-7.

3. Bartschat S, Mercer-Chalmers-Bender K, Beike J, Rothschild MA, Jubner M. Not only smoking is deadly: fatal ingestion of e-juice: a case report. Int J Legal Med 2015;129:481-6.

4. Schneider S, Diederich N, Appenzeller B, Schartz A, Lorang C, Wennig R. Internet suicide guidelines: report of a life-threatening poisoning using tobacco extract. J Emerg Med 2010;38:610-3.

5. Hagiya K, Mizutani T, Yasuda S, Kawano S. Nicotine poisoning due to intravenous injection of cigarette soakage. Hum Exp Toxicol 2010;29: 427-9.

6. Chen BC, Bright SB, Trivedi AR, Valento M. Death following intentional ingestion of e-liquid. Clin Toxicol (Phila) 2015;53:914-6.

7. Solarino B, Rosenbaum F, Riesselmann B, Buschmann CT, Tsokos M. Death due to ingestion of nicotine-containing solution: case report and review of the literature. Forensic Sci Int 2010;195:e19-22.

8. Kivrak T, Sunbul M, Durmus E, Dervisova R, Sari I, Yesildag O. Acute myocardial infarction due to liquid nicotine in a young man. Ther Adv Cardiovasc Dis 2014;8:32-4.

9. Kim JW, Baum CR. Liquid nicotine toxicity. Pediatr Emerg Care 2015;31: $517-21$.

10. Benowitz NL, Burbank AD. Cardiovascular toxicity of nicotine: implications for electronic cigarette use. Trends Cardiovasc Med 2016;26:515-23. 